
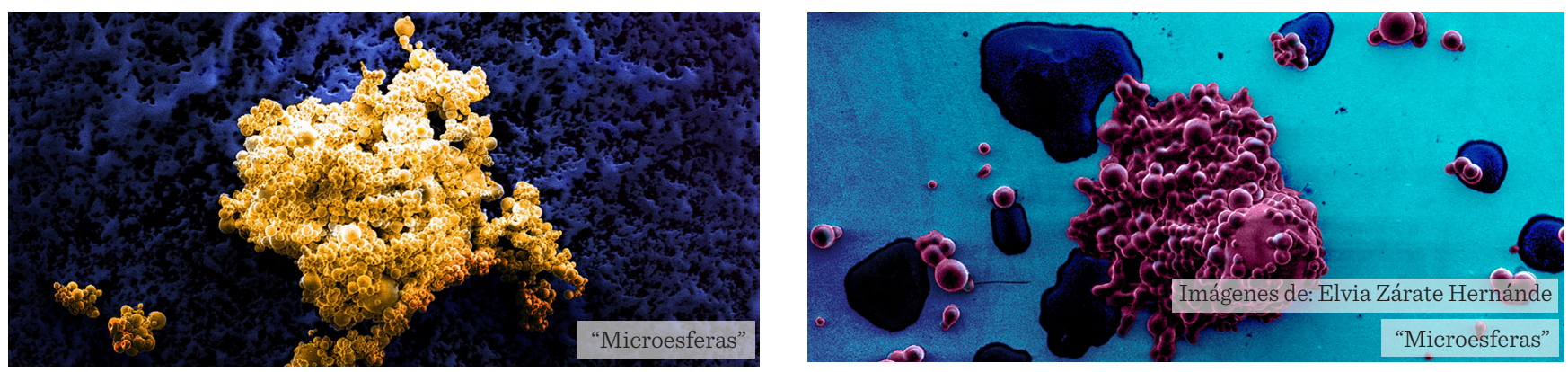

\title{
Microcápsulas y microesferas: una visión a la caracterización integral y aplicación para la liberación de medicamentos biotecnológicos Microcapsules and microspheres: A vision to integral characterization and applications for biotechnological drugs delivery
}

\author{
Elvia Zárate-Hernández, Rosa Alejandra Hernández-Esquivel, José Trinidad Pérez-Urizar*
}

\section{RESUMEN}

Los microacarreadores basados en microcápsulas y microesferas han sido ampliamente estudiados y ensayados para controlar la liberación de medicamentos biotecnológicos (MB), disminuyendo la dosificación o modificando la vía de administración. Los métodos para la obtención de microacarreadores, son complejos y variados, por lo que es necesario determinar los requisitos mínimos que debe cumplir el sistema. El objetivo de este trabajo fue establecer las principales características que deben ser evaluadas en los microacarreadores para garantizar que la actividad biológica de los medicamentos biotecnológicos permanezca intacta a través del proceso de microencapsulación $\mathrm{y}$, por lo tanto, que la seguridad del MB (desarrollo de reacciones inmunes) se mantenga inalterada. Las características a evaluar de un microacarreador deben describir las propiedades del material, tamaño y forma del sistema, carga de la partícula, funcionalidad, eficiencia de la microencapsulación y la cinética de liberación. Mientras que la integridad de los MB puede ser evaluada a partir de parámetros críticos de calidad: estructura y función biológica del MB, pureza del producto, presencia de agregados de alto peso molecular, estructura de orden superior y ensayos de actividad biológica. La caracterización de los microacarreadores debe enfocarse en la seguridad del biopolímero y proteínas ensayadas.

PALABRAS CLAVE: medicamento biotecnológico, microesferas, estabilidad proteica.

\section{ABSTRACT}

Microcarriers based on microcapsules and microspheres have been widely studied and tested to control the release of biotechnological drugs (BD), diminishing the dosage or modifying the route of administration. The methods for obtaining microcarriers are complex and varied, so it is necessary to determine the minimum characteristics with which the system must comply. The aim of this work was to establish the main characteristics that should be evaluated in the microcarriers in order to guarantee that the biological activity of biotechnological drugs remains intact through the microencapsulation process, and therefore the safety of the BD (development of immune reactions) remains unaltered. The characteristics of a microcarrier to be evaluated must describe the properties of the material, the size and shape of the system, the particle load, functionalization, the microencapsulation efficiency, and the kinetics of liberation. Whereas the integrity of BD can be evaluated by critical quality parameter such as: structure and biological function of the BD, product purity, presence of high molecular weight aggregations, higher order structure and biological activity tests. The characterization of the microcarriers must focus on the safety of the bipolymer and proteins tested.

KEYWORDS: biotechnology drug, microspheres, protein stability.

*Correspondencia: jpurizar@uasl.mx/Fecha de recepción: 9 de octubre de 2020/Fecha de aceptación: 12 de enero de 2021/Fecha de publicación: 30 de enero de 2021.

Universidad Autónoma de San Luis Potosí, Facultad de Ciencias Químicas, Laboratorio de Farmacología, Avenida Dr. Manuel Nava núm. 6, Zona Universitaria, San Luis Potosí, San Luis Potosí, México, C. P. 78210. 


\section{INTRODUCCIÓN}

En los últimos 30 años se han aprobado diversos medicamentos biotecnológicos (MB) para diferentes padecimientos, tales como diabetes, artritis o cáncer, por mencionar algunos (Schellekens, 2009; Bhawani y col., 2018). Con más de 8500 productos en desarrollo, esta industria fue valuada en $\$ 174.7$ billones de dólares en 2019, y para 2020 se proyectaba un crecimiento de \$248.7 billones de dólares (Agyei y col., 2017; Jiang y col., 2017; Informa, 2019).

En su mayoría, los MB se caracterizan por baja biodisponibilidad oral (menor al $2 \%$ ), tiempo de vida media corto en fluidos biológicos (aproximadamente de $30 \mathrm{~min}$ ) y una escasa absorción gastrointestinal. Estas características limitan la vía de administración y la confinan a la intravenosa, subcutánea e intramuscular (Bruno y col., 2013; Pawar y col., 2014; Pachioni-Vasconcelos y col., 2016).
Para mantener las concentraciones terapéuticas, los MB deben ser administrados varias veces al día, esquema que compromete el éxito terapéutico a largo plazo (Pawar y col., 2014; Pachioni-Vasconcelos y col., 2016). Para hacer frente a este problema, el uso de microacarreadores ha favorecido los sistemas de administración de fármacos, células y proteínas, disminuyendo los efectos colaterales asociados a los tratamientos y garantizando el éxito farmacológico (Pawar y col., 2014; Shah y Schwendeman, 2014). Los sistemas micrométricos están representados por polímeros, lípidos, metales y materiales inorgánicos (Tabla 1), los cuales pueden estar en el rango micro y nanométrico (Bilati y col., 2005; Bruno y col., 2013; Wang y col., 2013).

Cada sistema tiene peculiaridades únicas, sin embargo, los sistemas micrométricos ofrecen las siguientes ventajas: (i) protección al biofármaco de una rápida degradación y aclara-

Tabla 1. Ventajas y desventajas de los sistemas poliméricos.

Table 1. Advantages and disadvantages of polymeric systems.

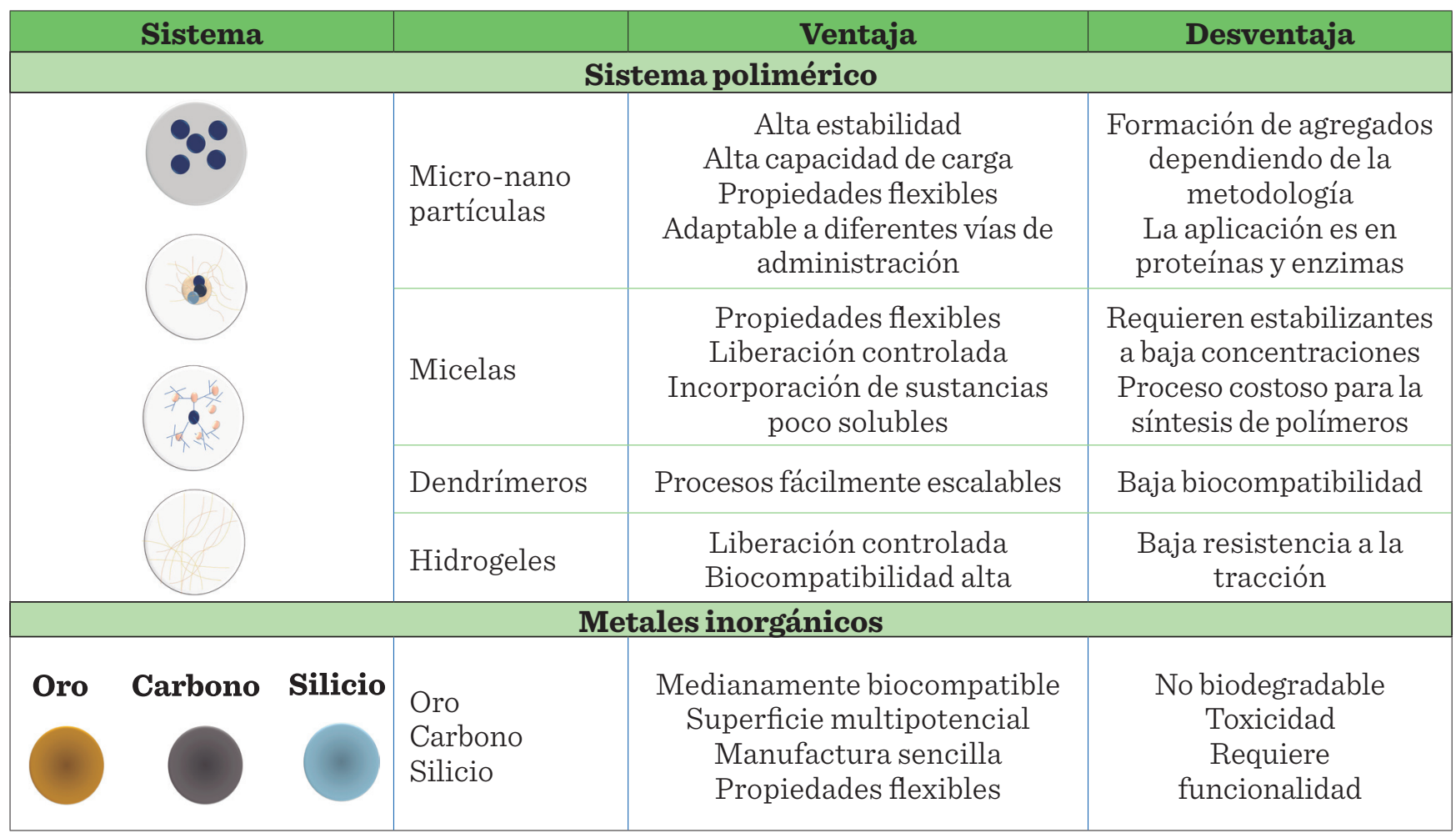

Fuente: modificado a partir de Bracho-Sanchez y col. (2016). 
miento después de su administración, así como de efectos ambientales de $\mathrm{pH}$, luz, temperatura y humedad; (ii) el efecto terapéutico puede prolongarse, a diferencia de la forma tradicional de administración y (iii) el perfil de liberación puede controlarse, dependiendo del diseño de la microesfera o microcápsula Figura 1 (Wang y col., 2013; Pachioni-Vasconcelos y col., 2016; Hu y col., 2017).

El objetivo de este trabajo fue destacar las principales características que deben ser evaluadas en el desarrollo de microacarreadores, enfatizando la seguridad de los medicamentos biotecnológicos, tras el proceso de microencapsulación, para el desarrollo de nuevas formas de administración.

Métodos de preparación de microcápsulas y microesferas a partir de biopolímeros

Las micropartículas tienen un diámetro medio de $1 \mu \mathrm{m}$ a $1000 \mu \mathrm{m}$ y pueden dividirse en microcápsulas o microesferas. El término "mi- crocápsula" hace referencia a un cuerpo esférico que recubre al principio activo, mientras que el término "microesfera" se refiere a una matriz homogénea, donde la sustancia activa está inmersa en el cuerpo de la esfera. Los métodos de obtención de microesferas y microcápsulas se categorizan en tres tipos ( Tabla 2): métodos químicos, fisicoquímicos y físico-mecánicos (Coelho y col., 2010; Jyothi y col., 2010; Singh y col., 2010; Wong y col., 2018). El material empleado debe ser biocompatible, de baja toxicidad y biodegradable en fluidos biológicos (Bracho-Sanchez y col., 2016). En este sentido, los polímeros hidrofílicos, como el alginato o quitosano, ofrecen una ventaja determinante al ser inmunotolerables y solubles en agua (McClements, 2018).

\section{Caracterización integral de microcápsulas y microesferas}

La caracterización integral de microesferas y micropartículas se resume en la Tabla 3 y está basada en la determinación de lo siguiente:

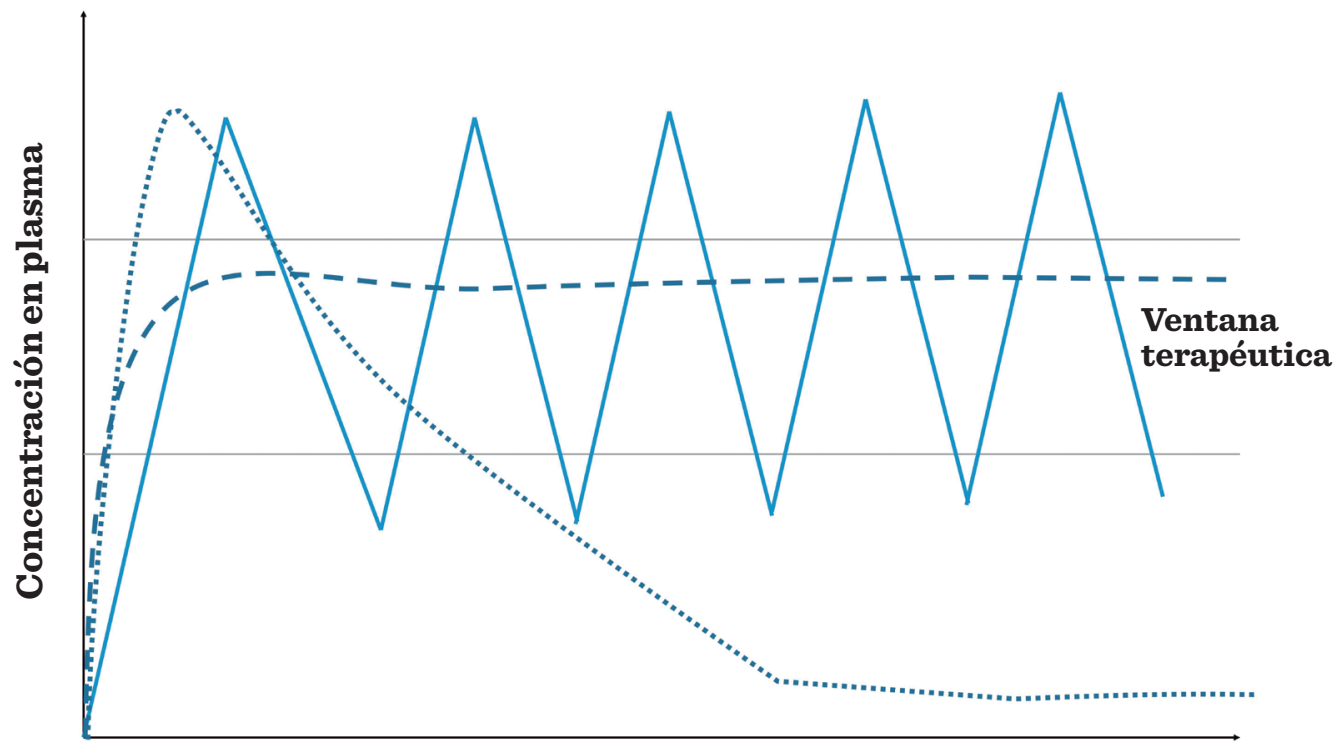

Tiempo

Fuente: modificado a partir de Wang y col. (2013).

Figura 1. Representación esquemática de la liberación de biofármaco en fluidos biológicos de forma tradicional (línea sólida), en comparación con la liberación de biofármaco en dosis repetidas (línea punteada) y a partir de un sistema en microesferas (línea segmentada).

Figure 1. Schematic representation of the release of biopharmaceutical into biological fluids in a traditional way (solid line), compared to the release of biopharma in repeated doses (dotted line) and from a system in microspheres (dotted line). 
Tabla 2. Métodos para la obtención de microcápsulas y microesferas.

Table 2. Methods for obtaining microcapsules and microspheres obtention.

\begin{tabular}{|c|c|c|c|c|c|c|}
\hline 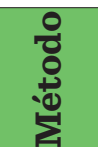 & $\begin{array}{l}\text { Polímero } \\
\text { empleado }\end{array}$ & $\begin{array}{c}\text { Material } \\
\text { encapsulado }\end{array}$ & $\begin{array}{l}\text { Factores de } \\
\text { proceso }\end{array}$ & $\underset{(\mu \mathrm{m})}{\operatorname{Tamaño}}$ & Aplicación & Referencias \\
\hline \multicolumn{7}{|c|}{ Métodos químicos } \\
\hline 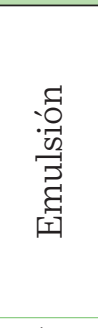 & $\begin{array}{c}\text { Polietilenglicol } \\
\text { Eextrinas } \\
\text { Quitosano } \\
\text { Alginato } \\
\text { Liposomas }\end{array}$ & $\begin{array}{l}\text { Súper óxido } \\
\text { dismutasa bovina } \\
\text { Albúmina } \\
\text { Sérica bovina } \\
\text { Metformina } \\
\text { Insulina } \\
\text { Cúrcuma }\end{array}$ & $\begin{array}{c}\text { Interfase } \\
\text { agua-aceite } \\
\text { Agitación } \\
\text { Reacción de } \\
\text { entrecruzamiento }\end{array}$ & $\begin{array}{c}0.5 \mathrm{a} \\
1000\end{array}$ & $\begin{array}{l}\text { Liberación } \\
\text { de fármacos }\end{array}$ & $\begin{array}{l}\text { Morita y col. } \\
\text { (2000); Yuan } \\
\text { y col. (2009); } \\
\text { Perry y } \\
\text { McClements } \\
\text { (2020); } \\
\text { Zheng y } \\
\text { McClements } \\
\text { (2020) }\end{array}$ \\
\hline 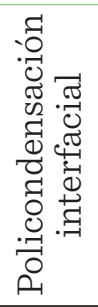 & - & $\begin{array}{c}\text { Albúmina sérica } \\
\text { bovina } \\
\text { Pigmentos }\end{array}$ & $\begin{array}{l}\text { Interfase de la } \\
\text { emulsión }\end{array}$ & $\begin{array}{l}0.5 \mathrm{a} \\
1000\end{array}$ & $\begin{array}{l}\text { Industria } \\
\text { alimenticia } \\
\text { Industria } \\
\text { farmacéutica }\end{array}$ & $\begin{array}{l}\text { Ozkan y col. } \\
\text { (2019) }\end{array}$ \\
\hline \multicolumn{7}{|c|}{ Métodos fisicoquímicos } \\
\hline 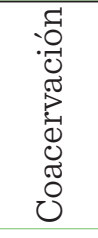 & $\begin{array}{l}\text { Goma } \\
\text { tragacanto } \\
\text { Gelatina } \\
\text { Goma arábica }\end{array}$ & Beta-caroteno & Aglomeración & 5 a 1000 & $\begin{array}{l}\text { Liberación de } \\
\text { fármacos }\end{array}$ & $\begin{array}{l}\text { Ozkan y col. } \\
\text { (2019) }\end{array}$ \\
\hline 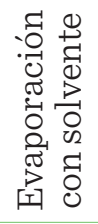 & $\begin{array}{c}\text { Ácido } \\
\text { poli(láctico- } \\
\text { co-glicólico) }\end{array}$ & Glucosa oxidasa & Aglomeración & $\begin{array}{l}0.5 \mathrm{a} \\
1000\end{array}$ & $\begin{array}{l}\text { Liberación de } \\
\text { fármacos }\end{array}$ & $\begin{array}{l}\text { Li y col. } \\
\text { (2000); } \\
\text { Ozkan y col. } \\
\text { (2019) }\end{array}$ \\
\hline 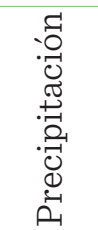 & Goma gelana & Proteínas & $\begin{array}{l}\text { Características } \\
\text { químicas de los } \\
\text { materiales }\end{array}$ & 5 a 1000 & $\begin{array}{l}\text { Liberación de } \\
\text { fármacos }\end{array}$ & $\begin{array}{l}\text { Coelho y col. } \\
\text { (2019) }\end{array}$ \\
\hline \multicolumn{7}{|c|}{ Métodos físico-mecánicos } \\
\hline 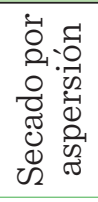 & $\begin{array}{l}\text { Alginato } \\
\text { Quitosano } \\
\text { Inulina } \\
\text { Maltodextrina }\end{array}$ & $\begin{array}{c}\text { Insulina } \\
\text { Jugo de arándano }\end{array}$ & Temperatura & 1 a 500 & $\begin{array}{l}\text { Industria } \\
\text { alimenticia } \\
\text { Industria } \\
\text { farmacéutica }\end{array}$ & $\begin{array}{l}\text { Yuan y col. } \\
\text { (2009); } \\
\text { Bowey y col. } \\
\quad(2013)\end{array}$ \\
\hline 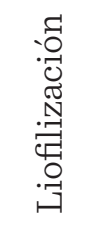 & $\begin{array}{c}\text { Celulosa } \\
\text { Maltodextrinas } \\
\text { Quitosano }\end{array}$ & $\begin{array}{l}\text { Albúmina sérica } \\
\text { bovina } \\
\text { Té verde } \\
\text { Toxoide tetánico }\end{array}$ & Sonicación & 20 a 500 & $\begin{array}{c}\text { Industria } \\
\text { farmacéutica } \\
\text { Industria } \\
\text { alimenticia }\end{array}$ & $\begin{array}{l}\text { Sánchez y } \\
\text { col. (1999); } \\
\text { Costantino y } \\
\text { col. (2000) }\end{array}$ \\
\hline 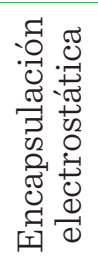 & $\begin{array}{l}\text { Alginato } \\
\text { Pectina }\end{array}$ & Extracto de cocoa & Capacitancia & 20 a 500 & $\begin{array}{l}\text { Industria } \\
\text { alimenticia }\end{array}$ & $\begin{array}{l}\text { Ozkan y col. } \\
\text { (2019) }\end{array}$ \\
\hline
\end{tabular}


Tabla 3. Principales técnicas de caracterización de microesferas y microcápsulas.

Table 3. Main techniques for characterization of microspheres and microcapsules.

\begin{tabular}{|c|c|c|c|c|}
\hline \multirow{2}{*}{$\begin{array}{l}\text { Parámetro } \\
\text { Material } \\
\text { (matriz) }\end{array}$} & \multicolumn{2}{|r|}{ Método } & \multirow{2}{*}{$\begin{array}{l}\text { Aplicación } \\
\text { Microesferas }\end{array}$} & \multirow{2}{*}{$\begin{array}{c}\text { Referencia } \\
\text { Chen y Zeng (2017) }\end{array}$} \\
\hline & NMR & $\begin{array}{c}\text { Resonancia magnética } \\
\text { nuclear }\end{array}$ & & \\
\hline & FTIR & $\begin{array}{l}\text { Espectroscopía de infrarrojo } \\
\text { con transformada de Fourier }\end{array}$ & Microesferas & Chen y Zeng (2017) \\
\hline & IR & Infrarrojo & Microesferas & Ribeiro y col. (2005) \\
\hline \multirow[t]{3}{*}{$\begin{array}{l}\text { Forma y tamaño } \\
\text { de partícula }\end{array}$} & TEM & $\begin{array}{l}\text { Microscopia electrónica de } \\
\text { trasmisión }\end{array}$ & $\begin{array}{c}\text { Albúmina } \\
\text { Interferón } \\
\text { Lisozima } \\
\text { Nanopartículas }\end{array}$ & $\begin{array}{c}\text { Zhou y col. (2008); } \\
\text { Nigen y col. (2010); } \\
\text { Bronze-Uhle y col. } \\
\text { (2017) }\end{array}$ \\
\hline & SEM & $\begin{array}{l}\text { Microscopia electrónica de } \\
\text { barrido }\end{array}$ & $\begin{array}{l}\text { Hormona de } \\
\text { crecimiento } \\
\text { Interferón } \\
\text { Eritropoyetina }\end{array}$ & $\begin{array}{c}\text { Zhou y col. (2008); } \\
\text { Jagtap y col. (2012); } \\
\text { Kang y col. (2014); } \\
\text { Ghasemi y col. (2019) }\end{array}$ \\
\hline & AFEM & $\begin{array}{l}\text { Microscopia de fuerza } \\
\text { atómica }\end{array}$ & Lisozima & Nigen y col. (2010) \\
\hline \multirow[t]{2}{*}{ Tamaño } & DLS & Radio hidrodinámico & $\begin{array}{l}\text { Insulina } \\
\text { Filgrastim }\end{array}$ & $\begin{array}{l}\text { Bowey y col. (2013); } \\
\text { Kiafar y col. (2016) }\end{array}$ \\
\hline & SPR & $\begin{array}{l}\text { Análisis de superficie de } \\
\text { plasmones }\end{array}$ & $\begin{array}{l}\text { Albúmina sérica } \\
\text { humana }\end{array}$ & Bhakta y col. (2015) \\
\hline Porosidad & MIP & $\begin{array}{l}\text { Potenciometría de intrusión } \\
\text { con mercurio o helio }\end{array}$ & Lisozima & $\begin{array}{c}\text { Reinhold y } \\
\text { Schwendeman (2013) }\end{array}$ \\
\hline \multirow[t]{2}{*}{$\begin{array}{l}\text { Propiedades } \\
\text { térmicas }\end{array}$} & DSC & $\begin{array}{l}\text { Calorimetría diferencial de } \\
\text { barrido }\end{array}$ & $\begin{array}{l}\text { Albúmina } \\
\text { sérica bovina } \\
\text { Eritropoyetina }\end{array}$ & $\begin{array}{l}\text { Kang y Singh (2003); } \\
\text { Jagtap y col. (2012) }\end{array}$ \\
\hline & TGA & Análisis termogravimétrico & Eritropoyetina & $\begin{array}{l}\text { Abdelhakim y col. } \\
\text { (2019); } \\
\text { Nguyen y col. (2020) }\end{array}$ \\
\hline $\begin{array}{l}\text { Eficacia de la } \\
\text { encapsulación/ } \\
\text { Cinética de } \\
\text { liberación }\end{array}$ & HPLC & Cromatografía de líquidos & $\begin{array}{l}\text { Filgrastim } \\
\text { Insulina }\end{array}$ & $\begin{array}{l}\text { Kiafar y col. (2016); } \\
\text { Agrawal y col. (2017) }\end{array}$ \\
\hline
\end{tabular}

i) propiedades del material, ii) tamaño de partícula, morfología, forma o estructura del cristal, iii) propiedades de superficie, como carga, funcionalidad, iv) eficiencia de la microencapsulación, v) cinética de liberación, vi) estabilidad del MB (Gill y col., 2010).

La forma de las partículas puede ser observada a partir de diversas microscopias, cuyo alcance depende de las necesidades y limitaciones propias de la metodología. Las microsco- pias más empleadas son microscopia electrónica de barrido (SEM, por sus siglas en inglés: Scanning Electron Microscopy), microscopia electrónica de transmisión (TEM, por sus siglas en inglés: Transmission Electron Microscopy), microscopia de fuerza atómica (AFEM, por sus siglas en inglés: Atomic Force Electroluminescence Microscopy), entre otras (Rokstad y col., 2014; Chogale y col., 2016). El radio hidrodinámico determina la vía por la cual podrá ser administrada la formulación, por lo 
que la técnica de dispersión dinámica de luz (DLS, por sus siglas en inglés: Dynamic Light Scattering) es comúnmente usada para este fin; la principal desventaja de este método es la interferencia que implican moléculas de grandes dimensiones, las cuales pueden sedimentar o formar agregados, comprometiendo la fiabilidad de los resultados (Andhariya y Burgess, 2016).

Las propiedades físicas de las microcápsulas y microesferas dependen de las propiedades térmicas de una sustancia y se analizan por calorimetría diferencial de barrido (DSC, por sus siglas en inglés: Differential Scanning Calorimetry) o termogravimetría (TGA, por sus siglas en inglés: Thermogravimetric Analysis), lo que permite determinar la temperatura y el flujo de calor asociado a las transiciones térmicas, como la temperatura de fusión o transición vítrea, así como pérdida de masa (Schick, 2009; Gill y col., 2010).

Las propiedades de superficie determinan el grado de biotolerabilidad de estas moléculas en el organismo receptor. Las metodologías empleadas son la espectroscopia fotoelectrónica de rayos X (XPS, por sus siglas en inglés: X-Ray Photoelectron Spectrometry), espectrometría de masas de iones secundarios de tiempo de vuelo (ToF-SIMS, por sus siglas en inglés: Timeof-Flight Secondary Ion Mass Spectrometry) y espectroscopía de infrarrojo con transformada de Fourier acoplado a reflectancia total atenuada (ATR-FTIR, por sus siglas en inglés: Attenuated Total Reflection-Fourier Transform InfraRed Spectroscopy).

La eficacia de atrapamiento puede evaluarse como i) la capacidad de encapsulación (CE), expresada en la relación de principio activo entre cantidad total de otros componentes de las micropartículas, o ii) la eficiencia de encapsulación (EE), que se define en términos de porcentaje o fracción del principio activo presente en el sistema y la cantidad del principio activo inicial (Paul y col., 2012; Kim y col., 2014). La eficacia de microencapsulación puede verse afectada por la concentración y peso mole- cular del biopolímero, el método de preparación, la solubilidad del fármaco y del polímero, las interacciones fármaco-polímero y la tasa de evaporación del solvente (Jyothi y col., 2010; Bale y col., 2016).

Evaluar la cinética de liberación de los microacarreadores determina la cantidad de medicamento disponible y el tiempo que tarda en hacerlo (Kim y col., 2014; Zhao y col., 2016). Los modelos de cinéticas se ajustan a: i) difusión, donde el mecanismo obedece a la velocidad con la que el líquido traspasa la membrana de polímero y la velocidad con la que el MB migra de la microesfera (Singh y col., 2010); ii) disolución, que depende del grado de solubilidad del polímero y del biofármaco en fluidos biológicos (Bittner y col., 1998; Singh y col., 2010), iii) erosión, proceso complejo que implica la hinchazón, difusión y disolución del sistema (Mohammed y col., 2017).

La caracterización integral de estos sistemas permite brindar seguridad y certeza a las formulaciones desarrolladas, al asegurar la biocompatibilidad con los tejidos. La biocompatibilidad es la habilidad que tiene el material de no inducir, directa o indirectamente, respuestas inflamatorias (Rokstad y col., 2014). Diversos estudios relacionan las propiedades fisicoquímicas, como peso del polímero, tamaño, forma, carga del sistema y vías de degradación, con el desarrollo de respuestas inmunes (Andorko y col., 2017). Por ejemplo, el tamaño de partícula es capaz de influenciar la distribución biológica y limita la vía de administración. Micropartículas diseñadas para liberación oral, con un diámetro medio de $11 \mu \mathrm{m}$, pueden localizarse en el intestino (placas de Peyer), mientras que las micropartículas de $5 \mu \mathrm{m}$ son trasladadas del intestino de rata al bazo por el sistema linfático (Benne y col., 2016; Savage y col., 2019; Alqahtani y col., 2020). La forma de la partícula influye en esta internalización, ya que el proceso depende de la interacción del sistema con células fagocíticas (células dendríticas); partículas esféricas favorecen este proceso, mientras que formas cilíndricas pueden decrecer la eficacia de la vía de trans- 
porte, partículas con otras formas favorecen la presentación de antígenos (Savage y col., 2019). Durante el metabolismo, las partículas son degradadas y esto favorece la liberación del principio activo, sin embargo, los productos de degradación pueden tener propiedades químicas diferentes al material inicial, por lo que pueden interaccionar de manera distinta con células del sistema inmune y los tejidos; estas interacciones no siempre son benéficas; los productos de degradación de materiales, como los poliésteres, pueden ser empleados como adyuvantes en la fabricación de vacunas (Andorko y col., 2017). El desarrollo de efectos no deseados se puede evaluar mediante ensayos in vitro, que precisan la interacción entre las micropartículas o nanopartículas con diferentes sistemas celulares. Los ensayos in vitro detallan posibles modificaciones en el crecimiento, reproducción y morfología celular (Li y col., 2015). En la Tabla 4 se exponen algunas de las principales pruebas in vitro para evaluar la citotoxicidad o viabilidad celular por parte de los materiales empleados para la fabricación de sistemas en escala nano o micrométrica.

Caracterización integral de estabilidad de biofármacos como proteínas terapéuticas Los medicamentos biotecnológicos dependen de su estructura para ejercer actividad biológica (eficacia), por lo que el sistema y método de microencapsulación deben asegurar la integridad del MB (Paul y col., 2012). La pérdida de esta estructura (calidad) podría llevar a la formación de productos de degradación que potencialmente minimizan el efecto terapéutico y modifican las propiedades farmacocinéticas y fármacodinámicas de los $\mathrm{MB}$, lo que conllevaría la presencia de diferentes efectos adversos y respuestas inmunes no deseadas (seguridad) (Butreddy y col., 2020; Schuster y col., 2020).

La integridad de una proteína farmacéutica puede ser estudiada en términos de estabilidad física y química. La degradación química incluye procesos de desamidación, isomerización, hidrólisis y oxidación, mientras que la degradación física es evidenciada por la formación de agregados que llevará invariablemente a un decaimiento en la actividad biológica (Kim y col., 2014).

La estabilidad se determina en parámetros críticos de calidad (Tabla 5), los cuales dependen de los atributos relacionados con la estructura y función biológica del MB. Los principales parámetros son, i) pureza del producto, ii) presencia de agregados de alto peso

Tabla 4. Principales ensayos in vitro para evaluar biocompatibilidad y citotoxicidad.

Table 4. Main in vitro trials to evaluate biocompatibility and cytotoxicity.

\begin{tabular}{|l|c|c|c|}
\hline \multicolumn{1}{|c|}{ Método } & Ensayo & Material & Bibliografía \\
\hline Apoptosis & Anexina V & Alginato & $\begin{array}{c}\text { Sáenz-del-Burgo-Martínezy } \\
\text { col. (2018) }\end{array}$ \\
\hline Hemolisis/necrosis & $\begin{array}{c}\text { Ensayo LDH } \\
\text { Ensayo MTT }\end{array}$ & Dextrano & $\begin{array}{c}\text { Ribeiro y col. (2013) } \\
\text { Khalkhaliy col. (2015); }\end{array}$ \\
\hline $\begin{array}{l}\text { Peroxidación de } \\
\text { lípidos (Especies } \\
\text { reactivas de oxígeno) }\end{array}$ & TBA & Nanocápsulas & Charão y col. (2019) \\
\hline $\begin{array}{l}\text { Citocompatibilidad } \\
\text { Viabilidad celular TBA }\end{array}$ & $\begin{array}{c}\text { Alginato } \\
\text { Nanopartículas }\end{array}$ & $\begin{array}{c}\text { Berger y col. (2017); } \\
\text { Calasans-Maia y col. (2019) }\end{array}$ \\
\hline
\end{tabular}

MTT = bromuro de 3-(4, 5- dimetiltiazol-2-ilo)-2, 5-difeniltetrazol; TBA = ácido tiobarbitúrico; LDH = lactato deshidrogenasa. 
Tabla 5. Principales técnicas de caracterización de proteínas.

Table 5. Main techniques for proteins characterization.

\begin{tabular}{|c|c|c|}
\hline Parámetro & Atributo & Método de control y/o caracterización \\
\hline Estructura primaria & $\begin{array}{c}\text { Peso de la cadena ligera y pesada } \\
\text { Masa intacta } \\
\text { Secuencia de aminoácidos }\end{array}$ & $\begin{array}{l}\text { LC-ESI-MS } \\
\text { LC-ESI-MS } \\
\text { Mapeo peptídico } \\
\text { Secuenciación }\end{array}$ \\
\hline $\begin{array}{l}\text { Estructura de orden } \\
\text { superior }\end{array}$ & Estructura secundaria y terciaria & $\begin{array}{c}\text { Dicroísmo circular } \\
\text { Intercambio de hidrógeno/deuterio } \\
\text { Cristalografía }\end{array}$ \\
\hline Otras modificaciones & $\begin{array}{c}\text { Oxidación } \\
\text { Deamidación }\end{array}$ & $\begin{array}{c}\text { RP-HPLC } \\
\text { Mapeo peptídico } \\
\text { Cromatografía de intercambio aniónico } \\
\text { Cromatografía de intercambio iónico } \\
\text { MP }\end{array}$ \\
\hline $\begin{array}{l}\text { Heterogeneidad de } \\
\text { tamaño }\end{array}$ & $\begin{array}{l}\text { Agregación } \\
\text { Fragmentación }\end{array}$ & $\begin{array}{c}\text { Cromatografía de exclusión por tamaño } \\
\text { Ultracentrifugación } \\
\text { Microscopía } \\
\text { Electroforesis capilar } \\
\text { SDS-PAGE } \\
\text { RP-HPLC }\end{array}$ \\
\hline Actividad biológica & $\begin{array}{c}\text { Unión blanco terapéutico a } \\
\text { receptor } \\
\text { Bioactividad }\end{array}$ & $\begin{array}{c}\text { Trasposomas } \\
\text { ELISA } \\
\text { Ensayos en modelos animales }\end{array}$ \\
\hline
\end{tabular}

LC-ESI-MS = cromatografía de líquidos acoplado a espectrometría de masas de ionización por electrospray; SDS-PAGE = electroforesis en una sola dimensión; RP-HPLC = cromatografía de líquidos de alta resolución en fase reversa.

Fuente: modificado a partir de Sekhon y Saluja (2011).

molecular, iii) la estructura de orden superior; iv) análisis de isoformas, v) ensayos de actividad biológica (Kim y col., 2014).

La determinación de agregados, partículas o fragmentos es un atributo crítico de calidad, el cual se relaciona directamente con la eficacia y seguridad de las proteínas terapéuticas (Wagh y col., 2018). La cromatografía de exclusión por tamaño (SEC-HPLC, por sus siglas en inglés: Size Exclusion Chromatography) y la electroforesis capilar (CE, por sus siglas en inglés: Capillary Electrophoresis) son las técnicas empleadas para analizar las variantes de peso (Kirchhoff y col., 2017; Wagh y col., 2018).
Las variantes de carga pueden ser seguidas mediante cromatografía aniónica (IEX, por sus siglas en inglés: Ionic Exchange Chromatography) o Electroforesis Capilar (EC); las diferencias en este parámetro afectan directamente los perfiles farmacocinéticos y farmacodinámicos (Zhao y col., 2016; Kirchhoff y col., 2017). La estructura de orden superior (secundaria y terciaria) puede ser determinada por cristalografía, dicroísmo circular (DC) o calorimetría diferencial de barrido (DCS).

Por otra parte, los modelos in vitro permiten describir el comportamiento del biofármaco liberado, mientras que los modelos in vivo de- 
tallan su biodisponibilidad en fluidos biológicos (Andhariya y Burgess, 2016), en tanto que los modelos animales deben asegurar: i) la evaluación a largo plazo en las formulaciones y ii) la medición de anticuerpos neutralizantes (Andhariya y Burgess, 2016).

La pérdida de estabilidad involucra respuestas celulares complejas, que dan como resultado la formación de citocinas pro-inflamatorias, reacciones de hipersensibilidad (reacciones alérgicas y anafilaxia) hasta la formación de anticuerpos neutralizantes (anticuerpos que inhiben la actividad biológica deseada del MB), además de la presencia de síntomas como dolor, anemia y fiebre, que darán como resultado modificaciones en las propiedades farmacocinéticas del MB (mayor aclaramiento renal y menor eficacia terapéutica) (Schellekens, 2002; Laptoš y Omersel, 2018).
Aplicaciones

Algunos microacarreadores empleados para la liberación de medicamentos biotecnológicos se resumen en la Tabla 6.

La insulina recombinante es el tratamiento de elección para combatir la diabetes mellitus, padecimiento que afecta a más de 439 millones de adultos en el mundo. En el trabajo publicado por Bowey y col. (2013) se caracterizaron las micropartículas de alginato e insulina obtenidas por la técnica de secado por aspersión. El resultado fueron micropartículas con un diámetro medio de $2.1 \pm 0.3 \mu \mathrm{m}$, de forma esférica y una eficiencia de carga del 38.2 $\pm 9.5 \%$. Se determinó por microscopia confocal la presencia de una distribución matricial de insulina en las micropartículas. El bioensayo in vitro, para la determinación de la potencia biológica, consistió en medir la fosforilación in-

- Tabla 6. Ejemplos de microformulaciones.

Table 6. Examples of microformulations.

\begin{tabular}{|c|c|c|c|}
\hline $\begin{array}{c}\text { Familia de } \\
\text { biofármacos }\end{array}$ & Proteína encapsulada & Matriz & Bibliografía \\
\hline Enzimas & $\begin{array}{c}\text { Heparina } \\
\text { Lisozima } \\
\text { Glucógeno fosforilasa } \\
\text { Fasudil } \\
\text { Alteplasa }\end{array}$ & $\begin{array}{c}\text { Dextranos } \\
\text { PSA } \\
\text { Microesferas porosas de } \\
\mathrm{Ca}_{2} \mathrm{CO}_{3}\end{array}$ & $\begin{array}{c}\text { Li y col. (2000); } \\
\text { Nguyen y O’Rear (2017); } \\
\text { Correa-Paz y col. (2019); } \\
\text { Han y col. (2020); } \\
\text { Mietzner y col. (2020) }\end{array}$ \\
\hline Hormonas & $\begin{array}{c}\text { Insulina } \\
\text { Somatropina } \\
\text { Albúmina } \\
\text { Filgrastim } \\
\text { Eritropoyetina } \\
\text { Interferón } \\
\text { Factor de crecimiento de } \\
\text { fibroblastos } \\
\text { Factor de coagulación }\end{array}$ & $\begin{array}{c}\text { Alginato } \\
\text { PLA } \\
\text { PLGA } \\
\text { Ácido hialurónico } \\
\text { Parafina } \\
\text { Liposomas } \\
\text { PLGA } \\
\text { PLA } \\
\text { PLGA } \\
\text { Gelatina } \\
\text { Alginato }\end{array}$ & $\begin{array}{c}\text { Bittner y col. (1998); } \\
\text { Katti y Krishnamurt (1999); } \\
\text { Diwan y Park (2003); } \\
\text { Hahn y col. (2004); } \\
\text { Yang y col. (2010); } \\
\text { Bowey y col. (2013); } \\
\text { Kiafar y col. (2016); } \\
\text { Kim y Kim (2016); } \\
\text { Gravastrand y col. (2017); } \\
\text { Lan y col. (2017); } \\
\text { Zhang y col. (2018); } \\
\text { Wang y col. (2019) }\end{array}$ \\
\hline Anticuerpos & $\begin{array}{c}\text { Anti TNF- } \alpha \\
\text { Antimesotelina } \\
\text { Anti p46 } \\
\text { Trastuzumab }\end{array}$ & $\begin{array}{l}\text { PLGA } \\
\text { Silicio poroso } \\
\text { Metacrilato }\end{array}$ & $\begin{array}{c}\text { Macura y col. (2013); } \\
\text { Marquette y col. (2014); } \\
\text { Horák y col. (2017); } \\
\text { Nifontova y col. (2019) }\end{array}$ \\
\hline
\end{tabular}


tracelular de la proteína quinasa B (PKB, por su siglas en inglés: Protein Kinase B) en la línea celular L6 de mioblasto (rata), donde la insulina liberada de las partículas presentó un $88 \pm 15 \%$ de actividad, demostrando que el secado por aspersión no impacta de forma significativa la estructura de la proteína.

Mientras que en el trabajo de Zhang y col. (2018) se diseñaron microesferas de finas capas de polivinil-sulfato y ácido poliláctico cargadas con insulina. El tamaño de las microesferas fue de $5.25 \pm 0.15 \mu \mathrm{m}$, con una eficiencia de carga del $33.7 \pm 0.19 \%$ y una liberación de $111.33 \pm 1.15 \mathrm{mg} / \mathrm{g}$ de insulina. El ensayo de eficacia preclínica in vi$v o$ consistió en probar el control glicémico de la formulación, en un modelo animal de diabetes tipo II, mediante la inducción del estado hiperglicémico en ratas hembra Sprague Dawley con la administración intraperitoneal de estreotozotocina $(55 \mathrm{mg} / \mathrm{kg})$. Se formaron seis grupos experimentales $(n=10)$ que recibieron tratamiento como se describe a continuación: grupo control (solución salina vía oral), grupo control negativo (200 U/kg insulina vía oral), grupo control positivo (5 U/kg insulina subcutánea) y tres grupos experimentales (100 U/kg, $200 \mathrm{U} / \mathrm{kg}$ y $300 \mathrm{U} / \mathrm{kg}$, todos vía oral). Se logró demostrar que los tratamientos ejercieron un control glucémico dependiente de la dosis, lo cual sugiere un método prometedor para la obtención de microesferas cargadas con MB (Zhang y col., 2018). Estos trabajos evidencian que la insulina presente en ambos sistemas se encuentra activa, a pesar de haberse obtenido por metodologías diferentes.

La osteoclasis mandíbular, asociada a medicamentos como los bisfosfonatos, genera problemas en pacientes medicados que requieren extracciones dentales, con la finalidad de disminuir los efectos colaterales de este tratamiento. Erten-Taysi y col. (2019) demostraron la exitosa fabricación de microesferas de quitosano cargadas con hormona paratiroidea humana por el método de gelación. Las microesferas cargadas tienen un tamaño promedio de $0.61 \mu \mathrm{m}$, con un valor de potencial z de (+) $18.5 \mathrm{mV}$. Se compararon varias formulaciones, pero las partículas con la proporción 3:1 de quitosano y hormona evidenciaron la eficacia de encapsulación más alta, con un 72.9 \pm $4.84 \%$ de hormona, porcentaje que decreció dramáticamente al modificar la relación. El estudio in vivo consistió en la observación del proceso necrótico. Después de la extracción de tres molares superiores en ratas hembra Sprague-Dawley de 6 semanas, las cuales fueron aleatorizadas en 4 grupos ( $n=12)$, como se describe a continuación: grupo control (la curación de los animales se permite sin la administración de tratamientos), grupo control negativo (se colocan $30 \mu \mathrm{L}$ de formulación con microcápsulas vacías en la región subperiostal) y 2 grupos experimentales. El grupo experimental 1 recibió hormona paratiroidea contenida en un hidrogel (0.05 $\mu \mathrm{g} / \mu \mathrm{L}$ por $3 \mathrm{~d})$, mientras que el grupo experimental 2 fue tratado con una sola administración de microesferas (0.233 $\mu \mathrm{g} / \mathrm{L}$ de hidrogel). En los días 10 y 21 después de la extracción dental, 6 animales de cada grupo fueron sacrificados para el análisis histomorfométrico. En ambos casos se observó un proceso necrótico, inferior en aquellos animales que recibieron una sola dosis de microesferas, a diferencia de aquellos que recibieron dosis repetidas de hormona paratiroidea (Erten-Taysi y col., 2019).

La hormona de crecimiento representa una alternativa de tratamiento para infantes con trastornos del crecimiento, falla renal o síndrome de Turner. Los problemas asociadas a las limitaciones de la proteína la confinan a la administración intramuscular (IM), dando como resultado inflamación en el sitio de administración y un pobre apego al tratamiento. En el trabajo presentado por Kang y col. (2014) se desarrollaron microesferas de dextranos con PLGA cargadas con hormona de crecimiento por el método de doble emulsion. La caracterización de estas microesferas evidenció partículas con un diámetro medio de $40 \mu \mathrm{m}$ a $100 \mu \mathrm{m}$. Mientras que la caracterización de la integridad de la proteína se realizó mediante cromatografía de exclusión por tamaño, para evaluar la presencia de fragmentos, y microscopia de dicroísmo circular, para determinar la estruc- 
tura de orden superior. La formulación de dextrano con PLGA y somatropina no desnaturalizó de forma significativa al $\mathrm{MB}$, al no decaer la presencia del monómero que conforma la proteína. La estructura de orden superior se determinó por comparación de la microscopia DC de una solución de proteína fresca y proteína contenida en el sistema de micropartículas. Los resultados no sugieren diferencia significativa en este parámetro, por lo que se asume que la proteína tendrá efecto biológico.

El ensayo de liberación in vivo consistió en la determinación de los niveles de hormona de crecimiento en plasma, en ratas hembra Sprague Dawley que fueron aleatorizadas en 3 grupos $(\mathrm{n}=5)$ que se detallan a continuación: grupo control (recibe hormona de crecimiento, $5 \mathrm{mg} / \mathrm{kg}$ vía subcutánea), grupo experimental 1 (microesferas de dextrano con PLGA, $5 \mathrm{mg} / \mathrm{kg}$ vía subcutánea) y grupo experimental 2 (microesferas sin la presencia de dextranos, $5 \mathrm{mg} / \mathrm{kg}$ vía subcutánea). Los días $1,2,3$, $4,5,6,7,8,10,12,15$ y 28 se tomó una muestra de sangre para la cuantificación de hormona de crecimiento, mediante un kit de ELISA (por sus siglas en inglés: Enzyme-Linked Immunosorbent Assay). Las curvas de concentración de hormona de crecimiento sérica versus el tiempo permitieron visualizar un aumento de concentración media después de la dosis. Los resultados evidenciaron una mayor concentración de hormona de crecimiento en suero y un perfil de liberación lento, hasta por $40 \mathrm{~d}$ en las microesferas de dextrano con PLGA, en comparación con las microesferas sin la presencia de dextrano, lo que sugiere un sistema altamente efectivo para la liberación controlada de hormona de crecimiento.

El modelo in vivo de eficacia consistió en evaluar la ganancia de peso en ratas macho Sprague Dawley hipofisectomizados de 6 semanas y tratados con un agente inmunosupresor (tacrolimus 50 g vía sc). Los animales de estudio fueron aleatorizados en 4 grupos $(n=5)$ para recibir tratamiento, de esta manera: grupo control negativo (solución salina), grupo con- trol positivo (solución de hormona de crecimiento, $6 \mathrm{mg} / \mathrm{kg}$ por $30 \mathrm{~d})$ y dos grupos experimentales: grupo experimental 1 (6 mg/kg de microesferas sin dextranos vía subcutánea) y grupo experimental $2(6 \mathrm{mg} / \mathrm{kg}$ de microesferas con dextranos vía subcutánea). La ganancia de peso se determinó cada $24 \mathrm{~h}$ por 40 d. Los resultados obtenidos con el modelo de eficacia abordado fueron consistentes con el modelo in vitro; el modelo in vivo confirmó un aumento de peso, superior del día 6 hasta el día 40 en aquellos animales tratados con microesferas de dextrano y PLGA, en comparación con el grupo control. Los resultados demostraron el desarrollo de una formulación de liberación controlada estable y con evidencia de actividad biológica (eficacia) de la hormona de crecimiento.

La microencapsulación o formación de microesferas de proteínas farmacéuticas, empleando biopolímeros para modificar la vía de administración, el régimen de dosificación o controlar la liberación de medicamentos biotecnológicos, se han estudiado arduamente, con la finalidad de caracterizar los procesos de fabricación de manera integral para asegurar que los MB no pierdan su estructura y por ende presenten menor actividad biológica y mayor inmunogenicidad, por lo que este trabajo describe las principales técnicas de caracterización de microesferas y proteínas farmacéuticas, para asegurar la viabilidad de estos sistemas de encapsulación.

\section{CONCLUSIONES}

El éxito de los microacarreadores como sistemas de liberación de medicamentos biotecnológicos está basado en la integridad de las proteínas farmacéuticas, a través del proceso de microencapsulación. Las nuevas estrategias para el desarrollo de microacarreadores se sustentan en la exploración y explotación de biopolímeros altamente biotolerables, de baja inmunogenicidad y fácil degradación en fluidos biológicos, así como en la optimización de los parámetros en cada método, para obtener sistemas con altos porcentajes de carga y de rendimiento, evitando la desnaturalización 
de las proteínas farmacéuticas. Las vías de degradación de biopolímeros y la falta de estabilidad de las proteínas podrían conducir al fracaso terapéutico y al desarrollo de reacciones inmunes no deseadas, como procesos inflama- torios y la aparición de anticuerpos neutralizantes. La caracterización integral de los microacarreadores debe ser gradual y enfocada en la seguridad tanto del biopolímero empleado como de las proteínas ensayadas.

\section{REFERENCIAS}

Abdelhakim, H. E., Coupe, A., Tuleu, C., Edirisinghe, M., and Craig, D. Q. M. (2019). Electrospinning optimization of eudragit E PO with and without chlorpheniramine maleate using a design of experiment approach. Molecular Pharmaceutics. 16(6): 2557-2568.

Agrawal, G. R., Wakte, P., and Shelke, S. (2017). Formulation, physicochemical characterization and in vitro evaluation of human insulin-loaded microspheres as potential oral carrier. Progress in Biomaterials. 6(3): 125-136.

Agyei, D., Ahmed, I., Akram, Z., Iqbal, H. M., and Danquah, M. K. (2017). Protein and peptide biopharmaceuticals: An overview. Protein and Peptide Lettters. 24(2): 94-101.

Alqahtani, M. S., Syed, R., and Alshehri, M. (2020). Size-dependent phagocytic uptake and immunogenicity of gliadin nanoparticles. Polymers. 12(11): 2576.

Andhariya, J. V. and Burgess, D. J. (2016). Recent advances in testing of microsphere drug delivery systems. Expert Opinion on Drug Delivery. 13(4): 593-608.

Andorko, J. I., Pineault, K. G., and Jewell, C. M. (2017). Impact of molecular weight on the intrinsic immunogenic activity of poly (beta amino esters). Journal of Biomedical Materials Research. Part A. 105(4): 1219-1229.

Bale, S., Khurana, A., Reddy, A. S., Singh, M., and Godugu, C. (2016). Overview on therapeutic applications of microparticulate drug delivery systems. Critical Reviews in Therapeutic Drug Carrier Systems. 33(4): 309-361.

Benne, N., van-Duijn, J., Kuiper, J., Jiskoot, W., and Slütter, B. (2016). Orchestrating immune responses: How size, shape and rigidity affect the immunogenicity of particulate vaccines. Journal of Controlled Release. 234: 124-134.

Berger, E., Breznan, D., Stals, S., Jasinghe, V. J., Gonçalves, D., Girard, D., ..., and Lavigne, C. (2017). Cytotoxicity assessment, inflammatory properties, and cellular uptake of Neutraplex lipid-based nanoparticles in THP-1 monocyte-derived macrophages. Nanobiomedicine. 4:1-14.

Bhakta, S., Seraji, M. S., Suib, S. L., and Rusling, J. F. (2015). Antibody-like biorecognition sites for proteins from surface imprinting on nanoparticles. ACS Applied Materials \& Interfaces. 7(51): 2819728206.

Bhawani, S. A., Husaini, A., Ahmad, F. B., and Asaruddin, M. R. (2018). Polymer based protein therapeutics. Current Protein and Peptide Science. 19(10): 972-982.

Bilati, U., Allemann, E., and Doelker, E. (2005). Strategic approaches for overcoming peptide and protein instability within biodegradable nano-and microparticles. European Journal of Pharmaceutics and Biopharmaceutics. 59(3): 375-388.

Bittner, B., Morlock, M., Koll, H., Winter, G., and Kissel, T. (1998). Recombinant human erythropoietin (rhEPO) loaded poly (lactide-co-glycolide) microspheres: influence of the encapsulation technique and polymer purity on microsphere characteristics. European Journal of Pharmaceutics and Biopharmaceutics. 45(3): 295-305.

Bowey, K., Swift, B. E., Flynn, L. E., and Neufeld, R. J. (2013). Characterization of biologically active insulin-loaded alginate microparticles prepared by spray drying. Drug Development and Industrial Pharmacy. 39(3): 457-465.

Bracho-Sanchez, E., Xia, C. Q., Clare-Salzler, M. J., and Keselowsky, B. G. (2016). Micro and nano material carriers for immunomodulation. American Journal of Transplantation. 16(12): 3362-3370.

Bronze-Uhle, E. S., Costa, B. C., Ximenes, V. F., and Lisboa-Filho, P. N. (2017). Synthetic nanoparticles of bovine serum albumin with entrapped salicylic acid. Nanotechnology Science and Applications. 10: 11-21.

Bruno, B. J., Miller, G. D., and Lim, C. S. (2013). Basics and recent advances in peptide and protein drug delivery. Therapeutic Delivery. 4(11): 1443-146\%.

Butreddy, A., Janga, K. Y., Ajjarapu, S., Sarabu, S., 
and Dudhipala, N. (2020). Instability of therapeutic proteins - An overview of stresses, stabilization mechanisms and analytical techniques involved in lyophilized proteins. International Journal of Biological Macromolecules. 167: 309-325.

Calasans-Maia, M. D., Barboza-Junior, C., Soriano-Souza, C. A., Alves, A., Uzeda, M., Martinez-Zelaya, and Rossi, A. M. (2019). Microspheres of alginate encapsulated minocycline-loaded nanocrystalline carbonated hydroxyapatite: therapeutic potential and effects on bone regeneration. International Journal of Nanomedicine. 14: 4559-4571.

Charão, M. F., Goethel, G., Brucker, N., Paese, K., Eifler-Lima, V. L., Pohlmann, A. R., and Garcia, S. C. (2019). Melatonin-loaded lipid-core nanocapsules protect against lipid peroxidation caused by paraquat through increased SOD expression in Caenorhabditis elegans. BMC Pharmacology \& Toxicology. 20(1): 1-7.

Chen, K. Y. and Zeng, S. Y. (2017). Preparation and characterization of quaternized chitosan coated alginate microspheres for blue dextran delivery. Polymers. 9(6): 210.

Chogale, M. M., Ghodake, V. N., and Patravale, V. B. (2016). Performance parameters and characterizations of nanocrystals: A brief review. Pharmaceutics. 8(3).

Coelho, J., Eusebio, D., Gomes, D., Frias, F., Passarinha, L. A., and Sousa, A. (2019). Biosynthesis and isolation of gellan polysaccharide to formulate microspheres for protein capture. Carbohydrate Polymers. 220: 236-246.

Coelho, J. F., Ferreira, P. C., Alves, P., Cordeiro, R., Fonseca, A. C., Gois, J. R., and Gil, M. H. (2010). Drug delivery systems: Advanced technologies potentially applicable in personalized treatments. EPMA Journal. 1(1): 164-209.

Correa-Paz, C., Navarro-Poupard, M. F., Polo, E., Rodríguez-Pérez, M., Taboada, P., Iglesias-Rey, R., and Pelaz, B. (2019). In vivo ultrasound-activated delivery of recombinant tissue plasminogen activator from the cavity of sub-micrometric capsules. Journal of Controlled Release. 308: 162-171.

Costantino, H. R., Firouzabadian, L., Hogeland, K., Wu, C., Beganski, C., Carrasquillo, K., and Tracy, M. A. (2000). Protein spray-freeze drying. Effect of atomization conditions on particle size and stability. Pharmaceutical Research.17(11): 1374-1383.

Diwan, M. and Park, T. G. (2003). Stabilization of recombinant interferon-alpha by pegylation for encapsulation in PLGA microspheres. International Journal of Pharmaceutics. 252(1-2): 111-122.

Erten-Taysi, A., Cevher, E., Sessevmez, M., Olgac, V., Mert-Taysi, N., and Atalay, B. (2019). The efficacy of sustained-release chitosan microspheres containing recombinant human parathyroid hormone on MRONJ. Brazilian Oral Research. 33: e086.

Ghasemi, R., Abdollahi, M., Emamgholi-Zadeh, E., Khodabakhshi, K., Badeli, A., Bagheri, H., and Hosseinkhani, S. (2019). mPEG-PLA and PLA-PEGPLA nanoparticles as new carriers for delivery of recombinant human Growth Hormone (rhGH). Scientific Reports. 8(1): 9854.

Gill, P., Moghadam, T. T., and Ranjbar, B. (2010). Differential scanning calorimetry techniques: applications in biology and nanoscience. Journal Biomolecular Techniques. 21(4): 167-193.

Gravastrand, C., Hamad, S., Fure, H., Steinkjer, B., Ryan, L., Oberholzer, J., and Rokstad, A. M. (2017). Alginate microbeads are coagulation compatible, while alginate microcapsules activate coagulation secondary to complement or directly through FXII. Acta Biomaterialia. 58: 158-167.

Hahn, S. K., Kim, S. J., Kim, M. J., and Kim, D. H. (2004). Characterization and in vivo study of sustained-release formulation of human growth hormone using sodium hyaluronate. Pharmaceutical Research.21(8): 1374-1381.

Han, P., Zhou, X., and You, C. (2020). Efficient multi-enzymes immobilized on porous microspheres for producing inositol from starch. Frontiers in Bioengineering and Biotechnology. 8: 380.

Horák, D., Hlídková, H., Kit, Y., Antonyuk, V., Myronovsky, S., and Stoika, R. (2017). Magnetic poly (2-hydroxyethyl methacrylate) microspheres for affinity purification of monospecific anti-p46 kDa/ Myo1C antibodies for early diagnosis of multiple sclerosis patients. Bioscience Reports. 37(2).

Hu, M., Guo, J., Yu, Y., Cao, L., and Xu, Y. (2017). Research advances of microencapsulation and its prospects in the petroleum industry. Materials. 10(4): 369.

Informa (2019). Pharmaprojects Pharma R\&D Annual Review 2019. [En línea]. Disponible en: https://pharmaintelligence.informa.com/ /media/ informa-shop-window/pharma/2019/files/whitepa pers/pharma-rd-review-2019-whitepaper.pdf. Fecha de consulta: 14 de marzo de 2020.

Jagtap, Y. M., Bhujbal, R. K., Ranade, A. N., and 
Ranpise, N. S. (2012). Effect of various polymers concentrations on physicochemical properties of floating microspheres. Indian Journal Pharmaceutical Sciences. 74(6): 512-520.

Jiang, M., Severson, K. A., Love, J. C., Madden, H., Swann, P., Zang, L., and Braatz, R. D. (2017). Opportunities and challenges of real-time release testing in biopharmaceutical manufacturing. Biotechnology and Bioengineering. 114(11): 2445-2456.

Jyothi, N. V., Prasanna, P. M., Sakarkar, S. N., Prabha, K. S., Ramaiah, P. S., and Srawan, G. Y. (2010). Microencapsulation techniques, factors influencing encapsulation efficiency. Journal of Microencapsulation.27(3): 187-197.

Kang, F. and Singh, J. (2003). Conformational stability of a model protein (bovine serum albumin) during primary emulsification process of PLGA microspheres synthesis. International Journal of Pharmaceutics. 260(1): 149-156.

Kang, J., Wu, F., Cai, Y., Xu, M., He, M., and Yuan, W. (2014). Development of Recombinant Human Growth Hormone (rhGH) sustained-release microspheres by a low temperature aqueous phase/aqueous phase emulsion method. European Journal of Pharmaceutical Sciences. 62:141-147.

Katti, D. and Krishnamurti, N. (1999). Preparation of albumin microspheres by an improved process. Journal of Microencapsulation.16(2): 231-242.

Khalkhali, M., Sadighian, S., Rostamizadeh, K., Khoeini, F., Naghibi, M., Bayat, N., ..., and Hamidi, M. (2015). Synthesis and characterization of dextran coated magnetite nanoparticles for diagnostics and therapy. BioImpacts: BI. 5(3): 141-150.

Kiafar, F., Siahi-Shadbad, M. R., and Valizadeh, H. (2016). Filgrastim (G-CSF) loaded liposomes: mathematical modeling and optimization of encapsulation efficiency and particle size. Bioimpacts. 6(4): 195-201.

Kim, N. A., Lim, D. G., Lim, J. Y., Kim, K. H., and Jeong, S. H. (2014). Comprehensive evaluation of etanercept stability in various concentrations with biophysical assessment. International Journal of Pharmaceutics. 460(1-2): 108-118.

Kim, S. J. and Kim, C. W. (2016). Development and Characterization of Sodium Hyaluronate Microparticle-Based Sustained Release Formulation of Recombinant Human Growth Hormone Prepared by Spray-Drying. Journal of Pharmaceutical Sciences. 105(2):613-622.
Kirchhoff, C. F., Wang, X. M., Conlon, H. D., Anderson, S., Ryan, A. M., and Bose, A. (2017). Biosimilars: key regulatory considerations and similarity assessment tools. Biotechnology and Bioengineering. 114(12): 2696-2705.

Lan, L., Tian, F. R., ZhuGe, D. L., ZhuGe, Q. C., Shen, B. X., Jin, B. H., ..., and Xu, H. L. (2017). Implantable porous gelatin microspheres sustained release of bFGF and improved its neuroprotective effect on rats after spinal cord injury. Plos One. 12(3): e0173814.

Laptoš, T. and Omersel, J. (2018). The importance of handling high-value biologicals: Physico-chemical instability and immunogenicity of monoclonal antibodies. Experimental and Therapeutic $\mathrm{Me}$ dicine. 15(4): 3161-3168.

Li, X., Zhang, Y., Yan, R., Jia, W., Yuan, M., Deng, X., and Huang, Z. (2000). Influence of process parameters on the protein stability encapsulated in poly-DL-lactidepoly (ethylene glycol) microspheres. Journal of Controlled Release. 68(1): 41-52.

Li, W., Zhou, J., and Xu, Y. (2015). Study of the in vitro cytotoxicity testing of medical devices. Biomedical Reports. 3(5): 617-620.

Macura, S. L., Steinbacher, J. L., MacPherson, M. B., Lathrop, M. J., Sayan, M., Hillegass, J. M., ..., and Mossman, B. T. (2013). Microspheres targeted with a mesothelin antibody and loaded with doxorubicin reduce tumor volume of human mesothe liomas in xenografts. BMC Cancer. 13(1): 400.

Marquette, S., Peerboom, C., Yates, A., Denis, L., Langer, I., Amighi, K., and Goole, J. (2014). Stability study of full-length antibody (anti-TNF alpha) loaded PLGA microspheres. International Journal of Pharmaceutics. 470(1-2): 41-50.

McClements, D. J. (2018). Encapsulation, protection, and delivery of bioactive proteins and peptides using nanoparticle and microparticle systems: A review. Advances in Colloid and Interface Science. 253:1-22.

Mietzner, R., Kade, C., Froemel, F., Pauly, D., Sta. mer, W. D., Ohlmann, A., and Breunig, M. (2020). Fasudil Loaded PLGA microspheres as potential intravitreal depot formulation for glaucoma therapy. Pharmaceutics. 12(8): 706.

Mohammed, M. A., Syeda, J. T. M., Wasan, K. M., and Wasan, E. K. (2017). An overview of chitosan nanoparticles and its application in non-parenteral drug delivery. Pharmaceutics. 9(4). 
Morita, T., Sakamura, Y., Horikiri, Y., Suzuki, T., and Yoshino, H. (2000). Protein encapsulation into biodegradable microspheres by a novel S/O/W emulsion method using poly (ethylene glycol) as a protein micronization adjuvant. Journal of Controlled Release. 69(3): 435-444.

Nguyen, C. T., Kim, C. R., Le, T. H., Koo, K. I., and Hwang, C. H. (2020). Magnetically guided targeted delivery of erythropoietin using magnetic nanoparticles: Proof of concept. Medicine. 99(19): e19972.

Nguyen, H. X. and O’Rear, E. A. (2017). Modified dextran, heparin-based triggered release microspheres for cardiovascular delivery of therapeutic drugs using protamine as a stimulus. Journal of Microencapsulation. 34(3): 299-307.

Nifontova, G., Ramos-Gomes, F., Baryshnikova, M., Alves, F., Nabiev, I., and Sukhanova, A. (2019). Cancer cell targeting with functionalized quantum dotencoded polyelectrolyte microcapsules. Frontiers in Chemistry. 7: 34.

Nigen, M., Gaillard, C., Croguennec, T., Madec, M. N., and Bouhallab, S. (2010). Dynamic and supramolecular organisation of alpha-lactalbumin/lysozyme microspheres: A microscopic study. Biophysical Chemistry. 146(1): 30-35.

Ozkan, G., Franco, P., De-Marco, I., Xiao, J., and Capanoglu, E. (2019). A review of microencapsulation methods for food antioxidants: Principles, advantages, drawbacks and applications. Food Chemistry. 272: 494-506.

Paul, M., Vieillard, V., Jaccoulet, E., and Astier, A. (2012). Long-term stability of diluted solutions of the monoclonal antibody rituximab. International Journal of Pharmaceutics. 436(1-2): 282-290.

Pachioni-Vasconcelos, J., Lopes, A. M., Apolinario, A. C., Valenzuela-Oses, J. K., Costa, J. S., Nascimento, L., ..., and Rangel-Yagui, C. de O. (2016). Nanostructures for protein drug delivery. Biomaterials Science. 4(2): 205218.

Pawar, V. K., Meher, J. G., Singh, Y., Chaurasia, M., Surendar-Reddy, B., and Chourasia, M. K. (2014). Targeting of gastrointestinal tract for amended delivery of protein/peptide therapeutics: strategies and industrial perspectives. Journal of Controlled Release. 196: 168-183.

Perry, S. L. and McClements, D. J. (2020). Recent advances in encapsulation, protection, and oral delivery of bioactive proteins and peptides using colloidal systems. Molecules 25(5): 1161.
Reinhold, S. E. and Schwendeman, S. P. (2013). Effect of polymer porosity on aqueous selfhealing encapsulation of proteins in PLGA microspheres. Macromolecular Bioscience.13(12): 1700-1710.

Ribeiro, A. J., Silva, C., Ferreira, D., and Veiga, F. (2005). Chitosan-reinforced alginate microspheres obtained through the emulsification/internal gelation technique. European Journal of Pharmaceutical Sciences. 25(1): 31-40.

Ribeiro, M. P., Morgado, P. I., Miguel, S. P., Coutinho, P., and Correia, I. J. (2013). Dextran-based hydrogel containing chitosan microparticles loaded with growth factors to be used in wound healing. Materials Science and Engineering: C. 33(5):29582966.

Rokstad, A. M., Lacik, I., de-Vos, P., and Strand, B. L. (2014). Advances in biocompatibility and physicochemical characterization of microspheres for cell encapsulation. Advanced Drug Delivery Reviews. 67: 111-130.

Sáenz-del-Burgo-Martínez, L., Ciriza-Astrain, J., Espona-Noguera, A., Xavier, I., Cabruja-Casas, E., Orive-Arroyo, G., ..., and Alvarez, M. (2018). 3D Printed porous polyamide macrocapsule combined with alginate microcapsules for safer cell-based therapies. Scientific Reports. 8(1): 8512.

Sánchez, A., Villamayor, B., Guo, Y., McIver, J., and Alonso, M. J. (1999). Formulation strategies for the stabilization of tetanus toxoid in poly (lactideco-glycolide) microspheres. International Journal of Pharmaceutics. 185(2): 255-266.

Savage, D. T., Hilt, J. Z., and Dziubla, T. D. (2019). In vitro methods for assessing nanoparticle toxicity. Methods in Molecular Biology. 1894:1-29.

Schellekens, H. (2002). Bioequivalence and the immunogenicity of biopharmaceuticals. Nature Reviews Drug Discovery. 1(6): 457-462.

Schellekens, H. (2009). Biosimilar therapeuticswhat do we need to consider? NDT Plus. 2(1): i27i36.

Schick, C. (2009). Differential scanning calorimetry (DSC) of semicrystalline polymers. Analytical and Bioanalytical Chemistry. 395(6): 1589-1611.

Schuster, J., Koulov, A., Mahler, H. C., Detampel, P., Huwyler, J., Singh, S., and Mathaes, R. (2020). In vivo stability of therapeutic proteins. Pharmaceutical Research. 37(2): 23.

Sekhon, B. S. and Saluja, V. (2011). Biosimilars: an overview. Biosimilars. 1: 1-11. 
Shah, R. B. and Schwendeman, S. P. (2014). A biomimetic approach to active selfmicroencapsulation of proteins in PLGA. Journal of Controlled Release. 196: 60-70.

Singh, M. N., Hemant, K. S., Ram, M., and Shivakumar, H. G. (2010). Microencapsulation: A promising technique for controlled drug delivery. Research in Pharmaceutical Sciences. 5(2): 65-777.

Wagh, A., Song, H., Zeng, M., Tao, L., and Das, T. K. (2018). Challenges and new frontiers in analytical characterization of antibody-drug conjugates. MAbs. 10(2): 222-243.

Wang, L., Liu, Y., Zhang, W., Chen, X., Yang, T., and Ma, G. (2013). Microspheres and microcapsules for protein delivery: strategies of drug activity retention. Current Pharmaceutical Design. 19(35): 63406352 .

Wang, X., Qi, F., Xing, H., Zhang, X., Lu, C., Zheng, J., and Ren, X. (2019). Uniform-sized insulin-loaded PLGA microspheres for improved early-stage periimplant bone regeneration. Drug Delivery. 26(1): 11781190.

Wong, C. Y., Al-Salami, H., and Dass, C. R. (2018). Microparticles, microcapsules and microspheres: A review of recent developments and prospects for oral delivery of insulin. International Journal of Pharmaceutics. 537(1-2): 223-244.

Yang, F., Song, F. L., Pan, Y. F., Wang, Z. Y., Yang, Y. Q., Zhao, Y. M., ..., and Zhang, Y. M. (2010). Preparation and characteristics of interferon-alpha poly(lactic-co-glycolic acid) microspheres. Journal of Microencapsulation. 27(2): 133-141.

Yuan, W., Wu, F., Guo, M., and Jin, T. (2009). Development of protein delivery microsphere system by a novel S/O/O/W multi-emulsion. European Journal of Pharmaceutical Sciences. 36(2-3):212-218.

Zhang, H., Wang, W., Li, H., Peng, Y., and Zhang, Z. (2018). Microspheres for the oral delivery of insulin: preparation, evaluation and hypoglycaemic effect in streptozotocin-induced diabetic rats. Drug Development and Industrial Pharmacy. 44(1): 109-115.

Zhao, Y. Y., Wang, N., Liu, W. H., Tao, W. J., Liu, L. L., and Shen, Z. D. (2016). Charge variants of an avastin biosimilar isolation, characterization, in vitro properties and pharmacokinetics in rat. Plos One.11(3): e0151874.

Zheng, B. and McClements, D. J. (2020). Formulation of more efficacious curcumin delivery systems using colloid science: enhanced solubility, sta- bility, and bioavailability. Molecules. 25(12): 2791.

Zhou, S., Sun, J., Sun, L., Dai, Y., Liu, L., Li, X., ..., and Zhang, Z. (2008). Preparation and characterization of interferon-loaded magnetic biodegradable microspheres. Journal of Biomedical Materials Research. Part B. 87(1): 189-196. 